\title{
A SuRVEy OF Visible Iris Recognition
}

\author{
Yali Song ${ }^{1}$, Yongzhong $\mathrm{He}^{1,2}$ and Jin Zhang ${ }^{1}$ \\ ${ }^{1}$ Beijing Key Laboratory of Security and Privacy in Intelligent Transportation, \\ Beijing Jiaotong University, China, Beijing \\ ${ }^{2}$ Science and Technology on Electronic Information Control Laboratory, \\ Chengdu, China
}

\begin{abstract}
In recent years, research on iris recognition in near-infrared has made great progress and achievements. However in many devices, such as most of the mobile phones, there is no nearinfrared device embedded. In order to use iris recognition in these devices, iris recognition in visible light is needed, but there are many problems to use visible iris recognition, including low recognition rate, poor robustness and so on. In this paper, we first clarified the challenges in visible iris recognition. We evaluate the effectiveness of three traditional iris recognition on iris collected from smart phones in visible light. The results show that traditional methods achieve accuracy not exceeding $60 \%$ at best. Then we summarize the recent advances in visible iris recognition in three aspects: iris image acquisition, iris preprocessing and iris feature extraction methods. In the end, we list future research directions in visible iris recognition.
\end{abstract}

\section{KEYWORDS}

visible iris recognition, mobile phones, iris image acquisition, feature extraction

\section{INTRODUCTION}

In recent years, smart phones have been widely used in various fields due to their portability and light weight. From the initial simple call, texting development to later receiving verification code, notepad, payment management and other applications, the hidden danger of user security caused by the excessive amount of data has become an important problem. Biometric recognition provides a feasible solution for user identity security authentication due to its stability and not easy to lose.

In all biometrics characteristics including fingerprint, palm print, gesture, iris and face, iris recognition is deemed to be the most trustworthy biometric identification technology because of its high stability, high recognition rate, and not easy to counterfeit., it has been widely used in finance, medical, security and other fields. With the gradual maturity of iris recognition technology, user acceptance has gradually improved, and the market space is huge.

Iris recognition based on smart phones embeds traditional iris recognition technology into smart phone devices, which can be divided into two categories, namely near-infrared iris image recognition and visible light iris image recognition. The former has a high cost due to needing to embed a near-infrared acquisition device in a mobile phone. However, because of its high recognition rate, it has been released on the market. For example, in May 2015, Fujitsu and 
Japanese operators jointly launched the first new Smartphone with iris recognition, which can unlock the phone through blinking; in August 2016, Samsung released the flagship machine Note7, equipped with iris recognition function, and later rushed out of the market due to the explosion; in March 2017, Samsung continued to release the new flagship S8/S8+ with iris recognition, which quickly unlocks the phone through the iris. The latter has obvious cost advantages because it does not require embedding near-infrared devices in mobile phones., but the recognition rate is low due to the lack of rich texture features of the visible iris image acquired by the ordinary camera.

This paper investigates and evaluates visible iris recognition, and its contributions are as follows:

1. Discussing the purpose and significance of visible iris recognition.

2. Clarifying the current challenges of visible iris recognition

3. Summarizing the recent advances in visible iris recognition

4. Discussing the future research directions in visible iris recognition

The rest of this paper is organized as follows. Section 2 clarified the challenges in visible iris recognition. Section 3 introduces some current iris databases briefly. In Section 4, we summarize some iris preprocessing methods. Section 5 describes several iris feature extraction techniques. Finally, section 6 briefly discusses the conclusions and future work.

\section{Preliminary Of Visible Iris Recognition}

This section will briefly describe the basis of iris recognition, mainly from the overall structure and challenges

\subsection{THE OVERALL ARCHITECTURE OF VISIBLE IRIS RECOGNITION}

Iris recognition is to determine people's identity by comparing the similarities between iris image features. The process of iris recognition technology generally involves the following four steps:

1. Iris image acquisition: The entire eye of a person is photographed using a specific camera device, and the captured image is transmitted to an image preprocessing software of the iris recognition system.

2. Image preprocessing: The acquired iris image is processed to meet the requirements of extracting iris features. It consists of three parts: iris positioning, iris image normalization and image enhancement.

3. Feature extraction: The feature points required for iris recognition are extracted from the iris image by a specific algorithm and then encoded.

4. The feature encoding of the iris texture is compared with the data in the database to achieve the purpose of identity recognition.

\subsection{The Challenges OF Visible Iris ReCOGNITION}

The iris can be divided into a light iris and a dark iris according to the color. Since the texture of the dark iris is difficult to be resolved under visible light, the traditional iris recognition is studied for the iris under the near infrared. However, visible iris recognition has gradually attracted 
people's interest due to the variety of imaging sensors and the advancement of recognition algorithms. Next, we will explain the challenges of visible iris recognition from two aspects: data acquisition and recognition algorithms.

\subsubsection{Data Acquisition}

For data collection, we use smartphone as a stand-alone collection device. Using the rear and front cameras of the mobile phone to collect iris images in indoor and outdoor environments. Figure 1 shows a small sample iris. From this we can see that the iris collected by the smartphone has problems such as low image resolution, more noise factors and not obvious texture features.

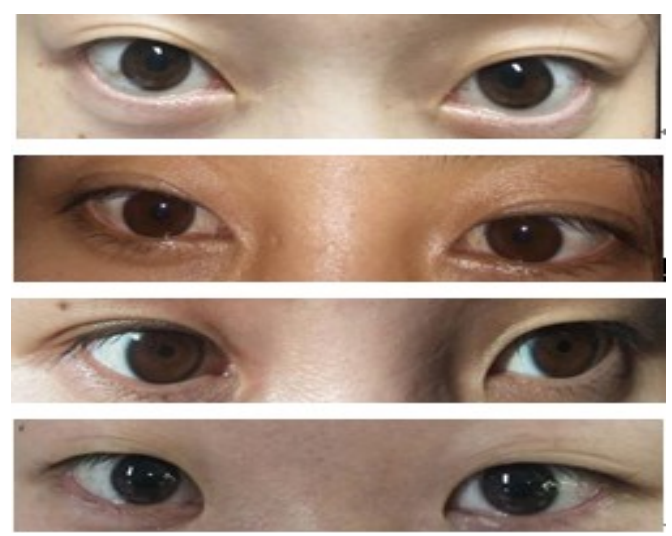

Figure 1. Sample images of iris

\subsubsection{Recognition Algorithms}

Due to the low quality of the visible iris collected by the smartphone, its iris texture information is less and difficult to extract. We use three traditional methods to evaluate the performance of visible iris captured by smartphones. According to the experiment, Table 1 lists the performance evaluation of the three methods. Figures 2 and Figures 3 depict the ROC curves for the three methods under outdoor conditions and indoor conditions. The results show that the recognition accuracy of the three traditional methods is the highest $60 \%$, which indicates that the traditional visible iris recognition method cannot extract the iris features well, and further research is needed.

Table 1. Performance evaluation

\begin{tabular}{|l|l|l|l|}
\hline \multicolumn{1}{|c|}{ Method } & RR & AUC & EER \\
\hline K-median clustering & 0.60 & 0.79 & 0.32 \\
\hline PCA & 0.56 & 0.76 & 0.40 \\
\hline Log-Gabor transformation & 0.43 & 0.72 & 0.64 \\
\hline
\end{tabular}




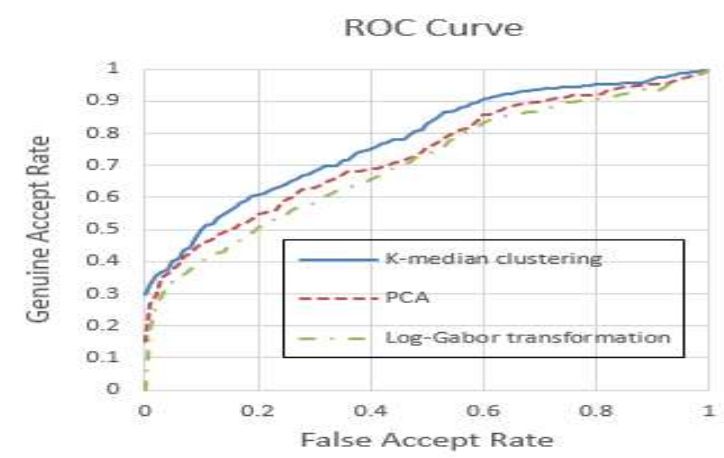

Figure 2. ROC curve in outdoor environment

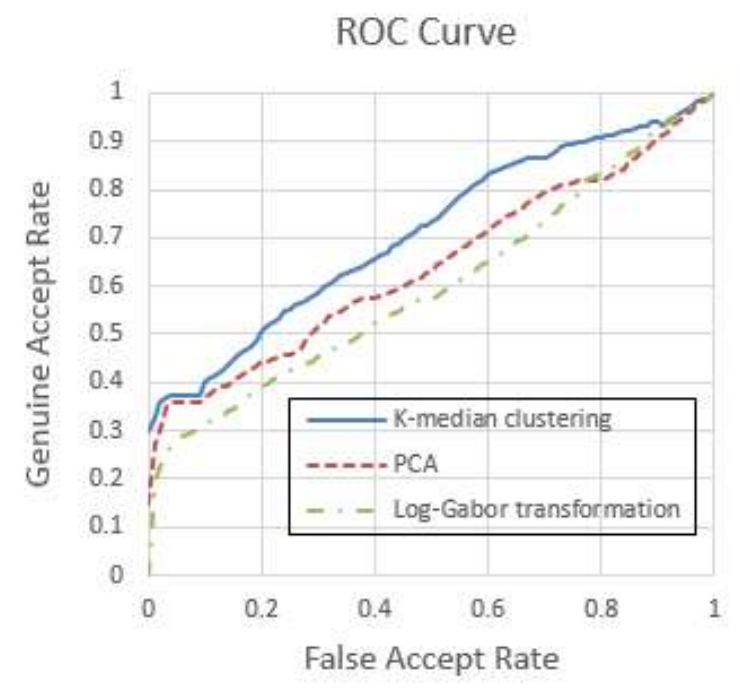

Figure 3. ROC curve in indoor environment

In view of some of the challenges listed above, we summarize the latest developments in visible iris recognition from three aspects: iris image acquisition, iris preprocessing and iris feature extraction. The specific content is shown in the following chapters.

\section{VISIBLE IRIS DATABASES}

Iris recognition performance is generally determined by the iris image quality and iris recognition methods. Thus, it is quite important to collect iris with high resolution. In this section, we will introduce some publicly available visible iris databases and some other visible iris databases collected by smartphones or light-field cameras.

\subsection{OPEN Visible IrIS DATA SETS}

There are some publicly available visible iris databases for iris images under visible light. UBIRIS.V2 [22] iris database is an iris image that is acquired over long distances in an indoor environment of natural light sources and artificial light sources. The subject is between 3-10 meters away from the collection device. The database includes 522 irises of 261 people, and a total of 11102 iris images are collected, which can be used for long-distance iris recognition 
under visible light. BIP Lab [4] also provides a visible iris dataset of 75 unique irises collected separately from indoor and outdoor using the front and rear cameras of the iPhone 5 and Samsung Galaxy S4 under two different illuminations. In addition, MICHE I and MICHE II also provide visible iris data sets for 4 samples under 8 different conditions under uncontrolled and different illumination using different mobile devices [5], Among them, MICHE I has 1600 pictures from 50 volunteers, while MICHE II has 3120 pictures from 75 volunteers.

\subsection{OTHER VISIBLE IRIS DATA SETS}

In addition to the above public datasets, some of the literature used their own iris datasets for experiments and performance comparisons with published datasets. Trokileewicz [1] used a mobile device to capture an image of 70 people's irises. The data was acquired by the iPhone 5S's rear camera and the flash was turned on indoors to ensure sufficient light when capturing images. The image quality exceeded the iris image of the near-infrared illumination, and finally 3192 images of different irises were collected. Kiran et al. [2] used two different smartphones, the iPhone 5S and Nokia Lumia, to collect iris images of different colors of volunteers from most Nordic countries under mixed illumination as data sets. The data was collected under the condition of semi-cooperative and unconstrained conditions, and 5 images of 28 people were collected indoors and outdoors, respectively, and a total of 560 iris images were collected. In addition, there is a patent that describes how iris collection should be performed and the issues that need to be noted during the acquisition process. In [3], it is mentioned that the light source and the eye's line of sight are usually adjusted to an angle of at least 30 degrees to direct the visible light onto the iris surface for iris feature collection. Table 2 summarizes some of the current visible iris data sets.

Table 2. Iris data sets

\begin{tabular}{|l|l|l|l|}
\hline Database & Subjects & Collection environment & Images \\
\hline UBIRIS .v1 & 241 & $\begin{array}{l}\text { 1)camera : Nikon E5700 } \\
\text { 2)focal length :8.9-71.2mm } \\
\text { 3)exposure time:1/30s } \\
\text { 4)ISO:200 }\end{array}$ & 1877 \\
\hline UBIRIS .v2 & 261 & $\begin{array}{l}\text { 1)camera : Canon EOS } \\
\text { 2)focal length :400mm } \\
\text { 3)exposure time:1/200 s } \\
\text { 4)ISO:1600 }\end{array}$ & 11102 \\
\hline UPOL & 62 & & 384 \\
\hline UBIPr & 344 & $\begin{array}{l}\text { 1)different postures } \\
\text { 2)different gaze } \\
\text { 3)different lighting }\end{array}$ & 10252 \\
\hline MICHE I & 50 & $\begin{array}{l}\text { 1)phone: iPhone5, } \\
\text { Samsung Galaxy S4 } \\
\text { 2)camera: front, rear } \\
\text { 3)environment: indoor, outdoor }\end{array}$ & 1600 \\
\hline MICHE II & 75 & $\begin{array}{l}\text { 1)phone: iPhone5, Nokia1020 } \\
\text { 2)camera: front, rear } \\
\text { 3)environment: indoor, outdoor }\end{array}$ & 3120 \\
\hline VSSIRIS & 28 & $\begin{array}{l}\text { 1)phone: iPhone5, } \\
\text { Samsung Galaxy S4 } \\
\text { 2)environment: Semi-cooperation, } \\
\text { unconstrained }\end{array}$ & 560 \\
\hline
\end{tabular}




\begin{tabular}{|l|l|l|l|}
\hline BDCP & 99 & camera :LG4000,CFAIRS & 1737 \\
\hline VISOB & 550 & $\begin{array}{l}\text { 1)phone: iPhone5, Oppo N1, Samsung Note 4, } \\
\text { 2) resolution: iPhone 720px, } \\
\text { Samsung and Oppo 1080px } \\
\text { 3) environment: conventional office, dim } \\
\text { office, natural light office }\end{array}$ & \\
\hline
\end{tabular}

\subsection{EVALUATION OF IRIS DATA SETS}

Based on the iris data sets listed above, we evaluated them as follows:

1. The current visible iris is mainly collected by cameras and smart phones. Different acquisition devices have different iris quality due to different camera configurations. For example, UBIRIS database and MICHE, VSSIRIS and VISOB databases have different iris resolutions. The number of irises collected by the same device is also different. The number of irises in the iris database MICHE and VSSIRIS collected for smartphones is quite different, and the scale is generally small.

2. Most mobile phone models currently collecting irises are iPhone or Samsung, and there is no attempt to collect them with other models and other resolutions.

3. Most of the iris database classifications are classified according to the collection equipment, and there is no classification based on other factors such as race, iris color and so on.

4. The current visible iris database can be used for long-distance iris recognition and lowquality iris recognition, in addition to visible iris recognition.

\section{Iris PREPRocessing}

After obtaining the iris image, the iris needs to be pretreated. Iris positioning is a key step in iris recognition, and its accuracy, robustness and positioning speed are extremely important. The purpose of iris localization is to determine the position of the inner and outer boundaries of the iris and the upper and lower eyelid boundaries in the image. In this section, we will discuss the iris localization scheme in iris recognition. The specific algorithms are as follows.

\subsection{INTEGRAL DifFERENTIAL OPERATOR METHOD}

Daugman [6-9] proposed using the integral differential operator method to globally search the center and radius to achieve iris localization. It is a geometric feature similar to the circle of the iris and the pupil, which can be achieved by detecting the circle. Its integral differential operator is defined as follows:

$$
\max _{\left(r, x_{p}, y_{0}\right)}\left|G_{\sigma}(r) * \frac{\partial}{\partial r} \underset{r, x_{0}, y_{0}}{\int} \frac{I(x, y) d s}{2 \pi r}\right|
$$


Where $\mathrm{I}(\mathrm{x}, \mathrm{y})$ is the acquired image, is the Gaussian function, and $\mathrm{r}$ is the search radius. It uses the first derivative to search and find the appropriate three parameters, that is, by changing the center and radius to search for the circle with the largest change of pixel value, iterating sequentially, and gradually reducing the amount of smoothing to achieve positioning. However, if the captured image contains more noise factors, the performance of the algorithm is greatly reduced.

\subsection{Hough Transform}

In 1996, Wildes [10] proposed the use of edge detection algorithm to detect the edge points of the image, and the hough transform was used to fit the position of the iris contour, which greatly improved its accuracy. Firstly, the edge map is generated by Canny edge detection. This method is implemented based on the edge detection of the gradient. And the set of inner and outer edge points of the iris is obtained by the threshold. Second, the hough transform is used to vote on all possible parameter sets (center and radius), and the parameter set corresponding to the largest vote is the detected circle. Its voting function is as follows:

$$
\begin{gathered}
H\left(x_{c}, y_{c}, r\right)=\sum_{j=1}^{n} h_{1}\left(x_{j}, y_{j}, x_{c}, y_{c}, r\right) \\
h_{1}\left(x_{j}, y_{j}, x_{c}, y_{c}, r\right)=\left\{\begin{array}{l}
1, \text { if } g\left(x_{j}, y_{j}, x_{c}, y_{c}, r\right)=0 \\
0, \text { if } g\left(x_{j}, y_{j}, x_{c}, y_{c}, r\right) \neq 0
\end{array}\right. \\
g\left(x_{j}, y_{j}, x_{c}, y_{c}, r\right)=\left(x_{j}-x_{c}\right)^{2}+\left(y_{j}-y_{c}\right)^{2}-r^{2}
\end{gathered}
$$

Where $\left(x_{j}, y_{j}\right)$ is all edge points, $h_{1}\left(x_{j}, y_{j}, x_{c}, y_{c}, r\right)$ is a function of whether or not to vote, $g\left(x_{j}, y_{j}, x_{c}, y_{c}, r\right)$ is a discriminant function of the circle. The parameter set that finally obtains the maximum value of $H\left(x_{c}, y_{c}, r\right)$ is the parameter sought.

\subsection{OTHER METHODS}

Jan [11] proposed an efficient non-circular iris contouring scheme that uses an integral differential operator, image grayscale intensity, pupil/iris geometry and adaptive threshold mixing to pinpoint the iris contour. Proença [12] proposed the use of color component analysis to determine the iris boundary by performing color channel analysis within the iris region of the image. Wang et al. [17] proposed using a repair method based on the Navier-Stokes (NS) equation to fill the reflection points, and using the possible boundary $(\mathrm{Pb})$ edge detection operator to initially detect the pupil edge to provide accurate positioning for low-quality iris images. Baek et al. [19] proposed an iris center localization algorithm based on the eyeball model to facilitate accurate positioning of the iris center under different head postures. Table 3 lists some of the positioning segmentation algorithms in the literature. 
Table 3. Iris localization and segmentation algorithm

\begin{tabular}{|l|l|l|l|}
\hline Reference & Method & Data sets & Accuracy \\
\hline Yingzi et al. [23] & ellipse fitting based on DLS & IUPUI & EER: $1.79 \%$ \\
\hline Daugman[6] & integral differential operator & & EER: $0.08 \%$ \\
\hline Wildes [10] & hough transform & & EER: $1.76 \%$ \\
\hline Jan [11] & $\begin{array}{l}\text { integral differential operator, } \\
\text { image gray intensity, } \\
\text { pupil/iris geometry } \\
\text { adaptive threshold }\end{array}$ & UBIRIS V1.0 & RR: $93.50 \%$ \\
\cline { 3 - 4 } & & MMU V1.0 & RR: $99.25 \%$ \\
\cline { 3 - 4 } & IITD V1.0 & RR: $99.46 \%$ \\
\hline Proença [12] & color component analysis & UBIRIS.v2 & EER: $5.02 \%$ \\
\hline Wang et al. [17] & Pb edge detection operator & $\begin{array}{l}\text { CASIA-Iris- } \\
\text { Thousand }\end{array}$ & EER: $1.82 \%$ \\
\hline Baek et al.[19] & $\begin{array}{l}\text { iris center positioning based on } \\
\text { eyeball model }\end{array}$ & Gi4e & RR: $81.4 \%$ \\
\cline { 3 - 4 } & merge active contour model & HPEG & RR: $88.6 \%$ \\
\hline Chen et al. [24] & K-means clustering & UBIRIS V1.0 & EER: 0.43 \\
\hline Sahmoud et al.[25] & RR: $98.76 \%$ \\
\hline
\end{tabular}

\subsection{Evaluation Of Iris Positioning}

For the above positioning method, the following evaluation was made:

1) They are mostly based on the circular geometry of the iris or combined with iris color analysis. As with the method of using only the iris shape or only the color analysis, the method of combining the two has a significantly better positioning effect. For example, the method in the Jan literature is better positioned than Proença.

2) The iris positioning effect is different on different datasets due to the different iris quality. Relatively speaking, the near-infrared iris dataset is better than the other datasets because of its better iris quality. Such as the CASIA-Iris-Thousand near-infrared data set and the UBIRIS.v2 visible data set.

3) Due to the long calculation time of the iris localization algorithm, the speed of positioning using the above method is not increased by an order of magnitude. Therefore, some faster methods can be studied for iris positioning, and positioning speed can be improved based on ensuring accuracy.

4) The above positioning method can be used for both near-infrared iris recognition and visible iris recognition. However, the positioning effect of the two must be different depending on the quality of the iris.

\section{IRIS FEATURE EXTRACTION}

Feature extraction refers to extracting unique feature points from the separated iris images by a certain algorithm and encoding them. A lot of literature has been introduced on the method of iris feature extraction. The specific method is as follows. 


\subsection{Ordinal Features And CNN Models}

Zhang et al. [14] proposed to measure the local iris texture by extracting the optimized ordinal measurement features, and then use the convolutional neural network to automatically learn the pairwise features to measure the correlation between the two irises. First, the ordinal feature selection is performed, and the normalized iris picture is divided into different areas using twoleaf and three-leaf ordinal filters, and different ordinal filters are applied to different regions. Select 15 regional features that can express texture information, reduce feature dimension and reduce processing time. After obtaining the OMs characteristics of the iris, the Hamming distance of the two feature templates is calculated. Secondly, using the paired CNN model to measure the correlation between the two irises, input two iris images of size $128 \times 128$, the intra-class pairs and the inter-class pairs are labeled as 1,0. The 64 pairs of filters in the first layer of convolutional layer are zero-filled the second and third layers are processed equally, and the dropout rate is set to 0.5 , using the dropout technique to prevent overfitting. The experiment finally achieved a better recognition effect of $0.8 \%$ EER.

\subsection{GeOMETRIC KeY}

Tan et al. [18] proposed feature extraction of long-distance iris images using geometric-keybased iris coding, and experiments in three published iris databases, compared with several other feature extraction methods, and finally proved that the proposed method has improved the recognition performance. First, the Log-Gabor transform is used to encode the global iris feature, which has the iris intensity of the iris region with less matching noise. Second, geometric information is used to provide efficient coding from local iris area pixels. Geometric keys (a set of coordinate pairs) are randomly generated and assigned to each topic, which uniquely defines the way the iris is coded, and the local iris feature is encoded based on the binary at the geometric key position. Then, configuring the geometry keys to resolve the proportional and rotational variations of the local iris area, and combining global iris coding with local iris coding to accommodate higher intra-injection imaging transformations. Finally, an effective match is made using the Hamming distance. The method uses three different databases to conduct experiments, and the error rates were $36.3 \%, 32.7 \%$ and $29.6 \%$.

\subsection{LOG-GABOR TRANSFORMATION}

Texture features are extracted from the radial and angular directions using a two-dimensional Log-Gabor filter. The Gabor function is as follows:

$$
g(x, y)=\frac{1}{2 \pi \delta_{x} \delta_{y}} \exp \left[-\left(\frac{x^{2}}{\delta_{x}^{2}}+\frac{x^{2}}{\delta_{y}^{2}}\right)\right] \times \cos [2 \pi f(x \cos \theta+y \cos \theta)]
$$

Where $f$ is the center frequency of the filter, $\theta$ is the filter direction, and $\left(\delta_{x}, \delta_{y}\right)$ is the Gaussian function standard deviation.

To achieve coverage of the filter in multiple directions, multiple filters are needed to show the discrepancy between different texture features. Its feature extraction formula is as follows: 


$$
F_{k j}(x, y)=H_{k j} * I(x, y)
$$

Where * is a convolution operation, $\mathrm{I}(\mathrm{x}, \mathrm{y})$ is the processed iris, $\mathrm{k}$ is the scale, and $\mathrm{j}$ is the direction.

\subsection{OTHER TECHNOLOGIES}

A lot of literature has been introduced on the method of iris feature extraction. Tan et al. [13] proposed using the phase information of the Zernike moment to encode the iris image and fuse it with the Gabor filtered result, and weight the code according to the information of the vulnerable bits. Kiran et al. [15] proposed a method for iris recognition by extracting iris features by multisegment depth sparse histograms combined with color channels. Raja et al. [2] proposed a new feature extraction method based on depth sparse filtering to obtain robust iris features. In [16], the strategy of iris and periocular information fusion is proposed to extract their respective features and fuse them, which can achieve cross-sensor iris recognition. Table 4 lists the feature extraction algorithms and their recognition effects in some literatures.

Table 4. Iris feature extraction algorithm

\begin{tabular}{|c|c|c|c|}
\hline Reference & Method & Data sets & Result \\
\hline \multirow[t]{3}{*}{ Tan et al. [18] } & \multirow[t]{3}{*}{ geometric key } & UBIRIS V2.0 & RR: $36.3 \%$ \\
\hline & & FRGC & RR:32.7\% \\
\hline & & CASIA.v4-distance & RR:29.6\% \\
\hline \multirow[t]{2}{*}{ Kiran. et al.[15] } & \multirow{2}{*}{$\begin{array}{l}\text { multi-segment depth sparse } \\
\text { histogram }\end{array}$} & MICHE-I & EER: $0.37 \%$ \\
\hline & & MICHE-II & GMR: $95 \%$ \\
\hline Raja et al. [2] & depth sparse filtering & VSSIRIS & EER:1.62\% \\
\hline \multirow[t]{3}{*}{ Tan et al.[13] } & \multirow{3}{*}{$\begin{array}{l}\text { zernike moment phase } \\
\text { characteristics }\end{array}$} & UBIRIS V2.0 & RR:54.3\% \\
\hline & & FRGC & RR: $32.7 \%$ \\
\hline & & CASIA.v4-distance & RR: $42.6 \%$ \\
\hline Zhang et al.[14] & $\begin{array}{l}\text { ordinal feature and CNN } \\
\text { model }\end{array}$ & & EER:1.2\% \\
\hline Radu et al, [26] & 2D Gabor wavelets & UBIRIS.v1 & EER:4.5\% \\
\hline Tsai [27] & LBP & UBIRIS V2.0 & ACC:92.5\% \\
\hline Raja et al.[28] & BSIF & & EER:1.05\% \\
\hline
\end{tabular}

\subsection{Evaluation Of Iris Feature Extraction}

For the above feature extraction method, the following evaluation was made:

1) The above feature extraction method can be used for both near-infrared iris recognition and visible iris recognition. However, due to the difference in the amount of iris texture information under near-infrared and visible light, the recognition effect under visible light is not as good as that under near-infrared (except for the iris acquired at a long distance).

2) The recognition effect of iris on different data sets is very different due to the difference in iris quality. For example, the recognition effect under UBIRIS and MICHE data sets is better than other data sets. 
3) At present, the average recognition rate of visible iris recognition is generally not high. Compared with the traditional recognition method, the use of depth sparse filtering or ordinal features and $\mathrm{CNN}$ model recognition accuracy is relatively good. Therefore, some more advanced feature extraction methods can be studied in order to better extract iris features.

\section{Discussion And Future Research Directions}

In recent years, due to the advantages of visible iris recognition, such as the use of convenient mobile phones, long-distance recognition, and iris obvious color discrimination, visible iris recognition based on smartphones is considered an important research topic. However, there are still some challenges for visible iris recognition.

1) The lower resolution of the iris image captured by the smartphone makes the texture information difficult to obtain.

2) Dark irises in Asians' visible light cause less texture information due to more melanin

3) Due to the low quality of the iris image, the accuracy of iris recognition using some traditional methods is low, and some more advanced methods must be studied to make it better to extract iris features.

In this paper, we investigated some databases on the identification of visible iris recognition, preprocessing methods and feature extraction methods. According to the survey, most of the visible iris recognition uses some open iris databases for experiments, but some of the literature use their own internal data sets for performance evaluation. The data set is small and not convincing. Secondly, most of the iris localization methods of the investigated literature are based on the circular geometry of the iris or combined with iris color analysis, and the average positioning effect is over $80 \%$. For the feature extraction method, most of the literatures have low recognition rate and high error rate, indicating that the current method still needs further improvement

In order to better promote the further development of visible iris recognition, in the future, our work can be carried out in the following aspects:

1) Large-scale visible iris database: The current visible iris datasets are few and the classification is not clear. In the future, irises can be collected to form new iris databases for different races and colors in a semi-cooperative environment.

2) Incorporating eye information: Since there is less information on the iris texture of the Chinese under visible light, some eye contour information can be added in the future to obtain more texture features.

3) Improvement of recognition method: Since the traditional recognition method cannot extract the characteristics of visible iris, some deep learning methods such as convolutional neural network can be used in the future.

\section{ACKNOWLEDGMENT}

This work was supported in part by the following grants: National key R\&D program of China No. 2017YFC0820100 


\section{REFERENCES}

[1] M. Trokielewicz, "Iris recognition with a database of iris images obtained in visible light using smartphone camera", IEEE International Conference on Identity Security and Behavior Analysis, pp. $1-6,2016$.

[2] KiranB. Raja, R. Raghavendra, V.K. Vemuri, C. Busch. Smartphone based visible iris recognition using deep sparse filtering. Pattern Recognit. Lett., 57 (2015), pp. 33-42

[3] Keith J. Hanna. Systems and methods for illuminating an iris with visible light for biometric acquisition. US9124798B2. 2011-05-17.

[4] http : //biplab.unisa.it/MICHE/database/

[5] M. De Marsico, M. Nappi, D. Riccio, H. Wechsler, "Mobile Iris Challenge Evaluation (MICHE)-I biometric iris dataset and protocols", Pattern Recognition Letters, vol. 57, pp. 17-23, 2015.

[6] J. Daugman. How iris recognition works. Proceedings of 2002 International Conference on Image Processing, Vol. 1, 2002

[7] J. Daugman. Biometric personal identification system based on iris analysis. United States Patent, Patent Number: 5,291,560, 1994.

[8] J. Daugman. High confidence visual recognition of persons by a test of statistical independence. IEEE Transactions on Pattern Analysis and Machine Intelligence, Vol. 15, No. 11, 1993.

[9] J. Daugman. Biometric decision landscapes. Technical Report No. TR482, University of Cambridge Computer Laboratory, 2000.

[10] R. Wildes. Iris recognition: an emerging biometric technology. Proceedings of the IEEE, Vol. 85, No. 9, 1997.

[11] Jan, F. (2016). Non-circular iris contours localization in the visible wavelength eye images. Computers and Electrical Engineering Journal, 62, 1-12.

[12] H. Proença. Iris recognition: on the segmentation of degraded images acquired in the visible wavelength. IEEE Trans. Pattern Anal. Mach. Intell., 32 (8) (2010), pp. 1502-1516

[13] Tan C W,Kumar A. Accurate iris recognition at a distance using stabilized iris encoding and Zernike moments phase features[J]. Image Processing, IEEE Transactions on, 2014, 23(9): 3962-3974.

[14] Zhang, Q., Li, H., Sun, Z., He, Z., Tan, T.: Exploring complementary features for iris recognition on mobile devices. In: International Conference on Biometrics (2016, to appear)

[15] K. Raja, R. Raghavendra, S. Venkatesh, C. Busch, Multi-patch deep sparse features for iris recognition in visible spectrum using collaborative subspace for robust verification, Pattern Recognit. Lett. Same Volume TBA.

[16] Xiao, L., Sun, Z., Tan, T.: Fusion of iris and periocular biometrics for cross-sensor identification. In: Zheng, W.-S., Sun, Z., Wang, Y., Chen, X., Yuen, P.C., Lai, J. (eds.) CCBR 2012. LNCS, vol. 7701, pp. 202-209.

[17] N. Wang, Q. Li, A.A.A. El-Latif, T. Zhang, X. Niu. Toward accurate localization and high recognition performance for noisy iris images. Multimed. Tools Appl. (2012), pp. 1-20 
[18] C.-W. Tan, A. Kumar. Efficient and Accurate At-a-Distance Iris Recognition Using Geometric KeyBased Iris Encoding. IEEE Transactions on Information Forensics and Security, 9 (9) (2014), pp. 1518-1526

[19] Baek, S.J., Choi, K.A., Ma, C., et al: 'Eyeball model-based iris center localization for visible imagebased eye-gaze tracking systems', IEEE Trans. Consum. Electron., 2013, 59, (2), pp. 415-421.

[20] Radman A, Zainal N, Ismail M. Efficient iris segmentation based on eyelid detection[J]. Journal of Engineering Science and Technology, 2013, 8(4): 399-405.

[21] Radu P, Sirlantzis K, Howells G, et al. A Versatile Iris Segmentation Algorithm[C]//BIOSIG.2011: 137-150.

[22] H. Proenca, S. Filipe, R. Santos, J. Oliveira, L. A. Alexandre, "The UBIRIS.v2: A database of visible wavelength iris images captured on-the-move and at-a-distance", IEEE Trans. Pattern Anal. Mach. Intell., vol. 32, no. 8, pp. 1529-1535, Aug. 2009.

[23] Y. Du, E. Arslanturk, Z. Zhou, C. Belcher.Video-based noncooperative iris image segmentation. IEEE Trans. Syst. Man Cybern. B, 41 (2011), pp. 64-74

[24] R. Chen, X.R. Lin, T.H. Ding. Iris segmentation for non-cooperative recognition systems. IET Image Process., 5 (2011), pp. 448-456.

[25] S.A. Sahmoud, I.S. Abuhaiba. Efficient iris segmentation method in unconstrained environments. Pattern Recogn., 46 (2013), pp. 3174-3185.

[26] P. Radu, K. Sirlantzis, W. Howells, F. Deravi, S. Hoque. Information fusion for unconstrained iris recognition. Int. J. Hybrid Inf. Technol., 4 (4) (2011), pp. 1-12

[27] Y. Tsai. A weighted approach to unconstrained iris recognition. Intl J. Comput. Inf. Syst. Control. Eng. (1) (2014), pp. 30-33

[28] K.B. Raja, R. Raghavendra, C. Busch. Binarized statistical features for improved iris and periocular recognition in visible spectrum. International Workshop on Biometrics and Forensics (IWBF) (2014), pp. 1-6 